\title{
Hollow Bulb Obturator for a Maxillectomy Patient - A Case Report
}

\author{
Dr.Sameera $\mathrm{K}^{1}$, Dr.Gilsa K Vasunni ${ }^{2}$, Dr. Ganesh Kamble ${ }^{3}$ \\ (Dept of Prosthodontics, Government Dental College, Calicut, India) \\ (Dept of Prosthodontics, Government Dental College, Calicut, India) \\ (Dept of Prosthodontics, Government Dental College, Calicut, India)
}

\begin{abstract}
Successful prosthetic rehabilitation in hemimaxillectomy patients require careful clinical and laboratory procedures to get an acceptable outcome. The main objectives of rehabilitation include restoration of aesthetic and cosmetic appearance of patient, restoration of function, protection of tissues, therapeutic and healing effect, psychological therapy and improving thequality of life.

This case report describes a clinical case of subtotal maxillectomy, which was rehabilitated successfully with a hollow bulb cast obturator.
\end{abstract}

Key words: Hemimaxillectomy, hollow bulb obturator, defect.

\section{Introduction}

Maxillofacial defects can be either congenital or acquired. Acquired defects are due to surgical resection of the tumors or due to trauma. As a result of this patients experiences unique alterations in the normal oral and craniofacial involvement, changes in the remaining natural teeth and tissue [1]. So the design must take consideration of the altered environment for the prosthesis support, stability and retention.

According to glossary of prosthodontics terms [2], obturator is defined as a prosthesis used to close a congenital or an acquired tissue opening primarily of hard palate and or contiguous alveolar structures. The name obturator is derived from the Latin verb "Obturare" which means to close.

The design of the obturator must be acceptable in appearance, should solve the problems in swallowing and speech and should exhibit only minimal movements under function [3] [4]. For providing this the obturator should:

1) Include maximum coverage of the edentulous ridge with in the movement capacity of the muscular attachments.

2) Use maximum engagement of the remaining teeth to help control retention and movement under function.

3) Facilitate correct placement of artificial teeth to provide tooth tissue contact during normal functional contacts.

This article describes the rehabilitation of a maxillectomy case with cast partial prosthesis having hollow bulb obturator.

\section{Case report}

A 27 year old male patient reported to the department with the chief complaint of missing teeth and difficulty in speech and deglutition (Fig.1). He had undergone surgery for squamous cell carcinoma of maxilla 6 months back. Intraoral examination of the patient showed subtotal maxillectomy of the right side which could be categorized under Aramany class II situation [5] [6]. The tooth missing were 13, 14,15,16,17. There was gingival recession on 12 but no mobility or pockets were present (Fig.2, Fig.3). Patient showed difficulty in mandibular movements with reduced mouth opening.

\section{Treatment plan}

The treatment objective was to give a screw gag initially for 8 weeks to get adequate mouth opening for performing the clinical steps .And then to rehabilitate the patient with a definitive hollow bulb cast obturator considering support, stability, retention and longevity.In the present case, support was provided by the remaining posterior teeth and palatal tissues for the prosthesis. Retention was achieved by use of circumferential clasp on first premolars and embrasure clasp on molars and partially by anatomic undercuts.

After getting the adequate mouth opening a perforated stock tray was selected for preliminary impression. A gauze piece was packed in the defect and floss was tied to it for retrieval after the impression was made. Impression was made with irreversible hydrocolloid (Zhermackneocolloid) (Fig.4) .Impression was boxed and then poured in type II stone (Kalabhai Karson Pvt Ltd, Mumbai).

The cast was then surveyed. It was designed to give a palatal strap framework modified with additional rests on cingulum of canines. Rest seat preparation was done on distoocclusal surface of first premolar and first 
molar and mesioocclusal surface of second molar. I bar clasp was given engaging right central incisor in addition to lateral incisor, considering the prognosis of lateral incisor.

Special tray was then fabricated with self-cure resin with spacers and stops on dentulous portion (premolar and molar).Border molding was done to record the soft tissues surrounding the defect using green stick impression compound(DPI pinnacle tracing sticks). And final impression was made with medium body elastomer (Reprosil, Dentsply) (Fig.5).

Cast partial framework was fabricated and tried in the patient's mouth for fit (Fig.6). Self-cure resin was applied on the mesh portion of the defect side, border molding was done and defect was reproduced with putty elastomer and wash impression made with light body elastomer impression material. An altered cast was then made. Jaw relation was then recorded on the permanent metal frame and try in done (Fig.7).

\section{Laboratory procedure}

The polished trial denture was invested in the lower half of the denture flask. Counter pour was made. Then after dewaxing one thickness of wax sheet was adapted only over the defect portion and one thickness also on opposite to the defect in the counter of the denture flask. A small strip of wax was removed from three portions: one from right, second from left and third from the depth of the defect (Fig.8). After applying cold mould seal a thin sheet of self-cure resin was prepared and adapted over the wax on both defect and opposite side.

When the denture flask was closed the two portions joined to form a hollow bulb inside. When the heat cure resin was packed the self-cure hollow bulb was embedded in the defect. For proper seating of the hollow bulb the three stops were used. Thus in the final processed obturator with the exception of the three stops, all other areas of the bulb will be covered with heat cured resin The prosthesis was then processed and polished(Fig.9,Fig.10).

During the time of insertion the patient was given the necessary instructions for use. Recall check-up was done after 24 hours, 1 week, first ,third and sixth months with satisfactory results (Fig.11).

\section{Discussion}

A common feature among all removable obturator prosthesis is that the framework design should be dictated by basic prosthodontics principles of design. These include broad stress distribution, cross arch stabilization with use of a rigid major connector, stabilizing and retaining components at locations within the arch to best minimize dislodging functional forces and replacement tooth positions that optimize prosthesis stability and functional needs[7] .

In the present case, the defect could be categorized under Aramany classification II. The metal framework was designed with required components. The regular palatal strap major connector was modified with additional indirect retention from cingulum of anterior teeth in order to achieve more support from the palate, as the defect was large. The metal framework provided good retention, support and stability. Further, the thermal conductivity of the metal made it sensitive to the temperature changes and the patient showed better functional acceptance to the prosthesis [8].

A hollow bulb obturator allows fabrication of a light weight prosthesis, which is readily accepted by the patient while effectively extending into the defective areas [9] [10]. It reduces the weight, is more hygienic, easy to fabricate, and increase speech clarity. It also prevents fluid and food collection unlike an open bulb obturator which is unhygienic, foul smelling and unpleasant for the patient [11].

\section{Conclusion}

In this case a cast obturator with a hollow bulb was fabricated for a defect of palate. The prosthesis rehabilitated the patient in terms of function by providing better masticatory efficiency, phonetics by adding resonance to the voice hence improving the clarity of speech and also improved the esthetics of the patient. The use of a hollow bulb design improved the comfort of the patient by decreasing the weight of the prosthesis. The prosthesis improved the appearance, beauty, function and comfort of the patient.

\section{References}

[1] Beumer .J, Curtis .T.A, Firtel D N, Maxillofacial rehabilitation. (St.Louis:Mosby ,1979) 188-243.

[2] GPT-8.The academy of prosthodontics. J Prosthet Dent, 2005; 94:56.

[3] Desjardins R.Obturator prosthesis design for acquired maxillary defect. J Prosthet Dent 1978, 39:424-435.

[4] Taylor T, Gerrow J, Brudvik J. Resin bonded components for maxillofacial prosthesis construction. A clinical trial. J Prosthet Dent.1988; 59; 334-339.

[5] Aramany MA. Basic principles of obturator design for partially edentulous patients. Part I: Classification. J prosthet dent 2001, 86(6), 559-561.

[6] Aramany MA. Basic principles of obturator design for partially edentulous patients. Part II: Design principles. J prosthet dent 2001, 86(6), 562-568.

[7] Gregory RP, Greggory ET, and Arthur OR. Prosthodontic principles in the framework design of maxillary obturator prostheses. $J$ 
Prosthet Dent 2005; 93:405-411.

[8] Mccrackens. Removable Partial Prosthodontics. (11th ed, Mosby Elsevier) 79-107.

[9] Chalian. Maxillofacial prosthetics (Elsevier publication).

[10] Habib BH, Carl F. Driscoll. Fabrication of a closed hollow obturator. J Prosthet Dent 2004; 91:383-5.

[11] McAndrew KS, Rothenberger S, Minsley GE. An innovative investment method for the fabrication of a closed hollow obturator prosthesis. J Prosthet Dent 1998; 80:129-32.

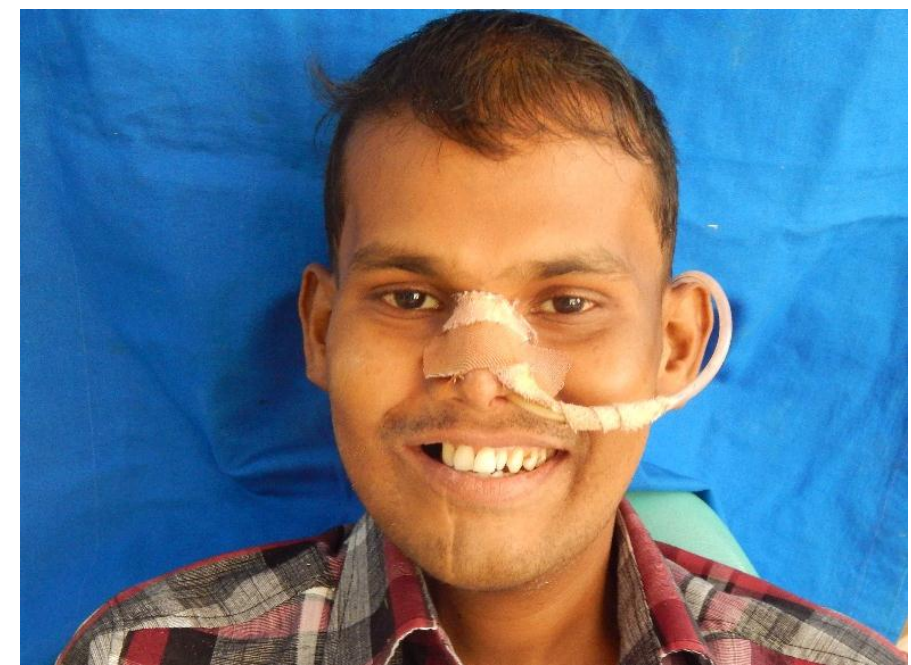

Fig.1Preoperative photograph

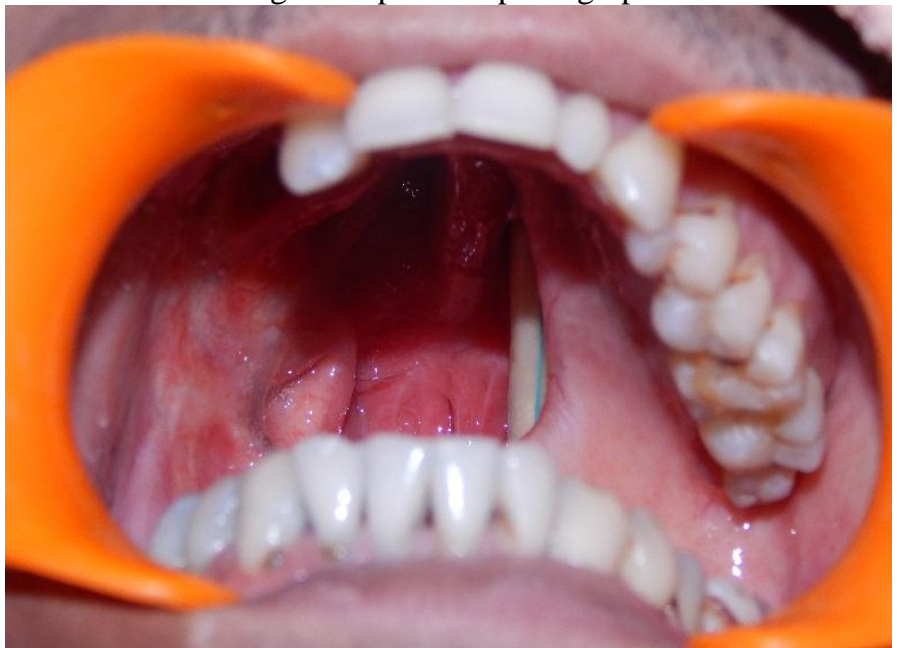

Fig.2 Intra oral view (defect)

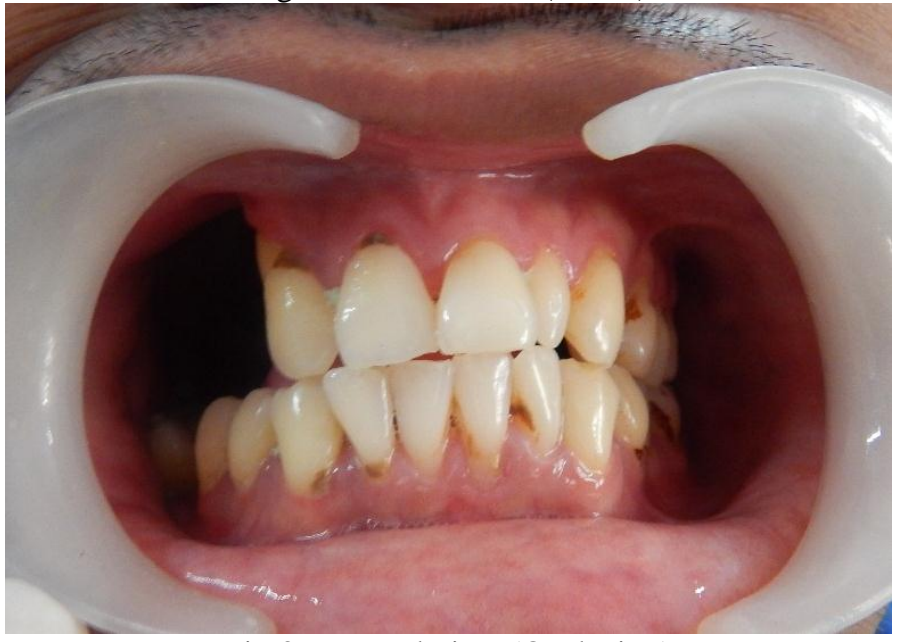

Fig.3 Intraoral view (Occlusion) 


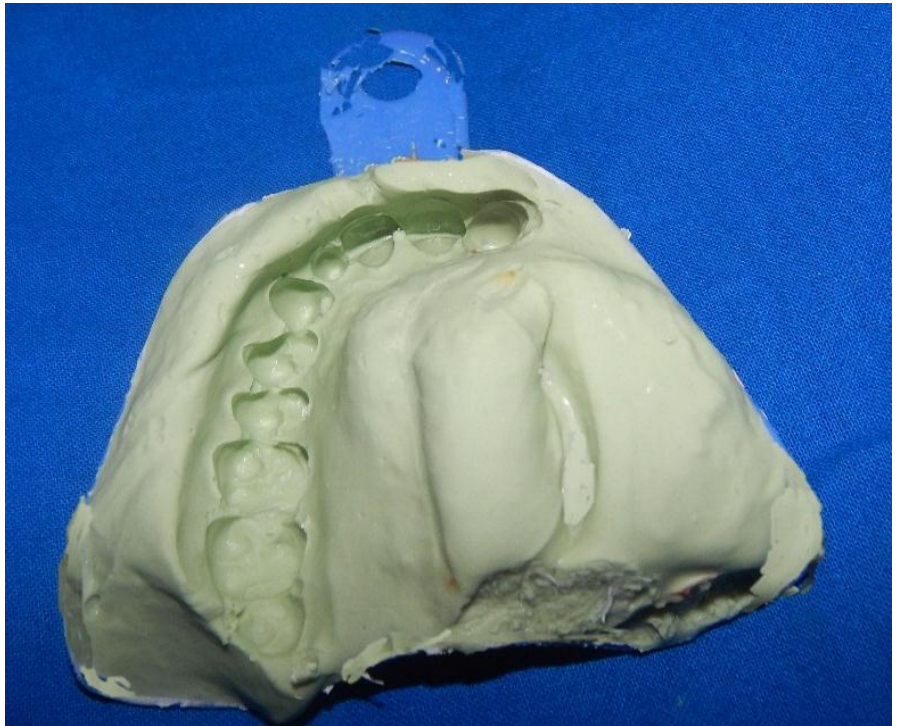

Fig.4 Preliminary impression

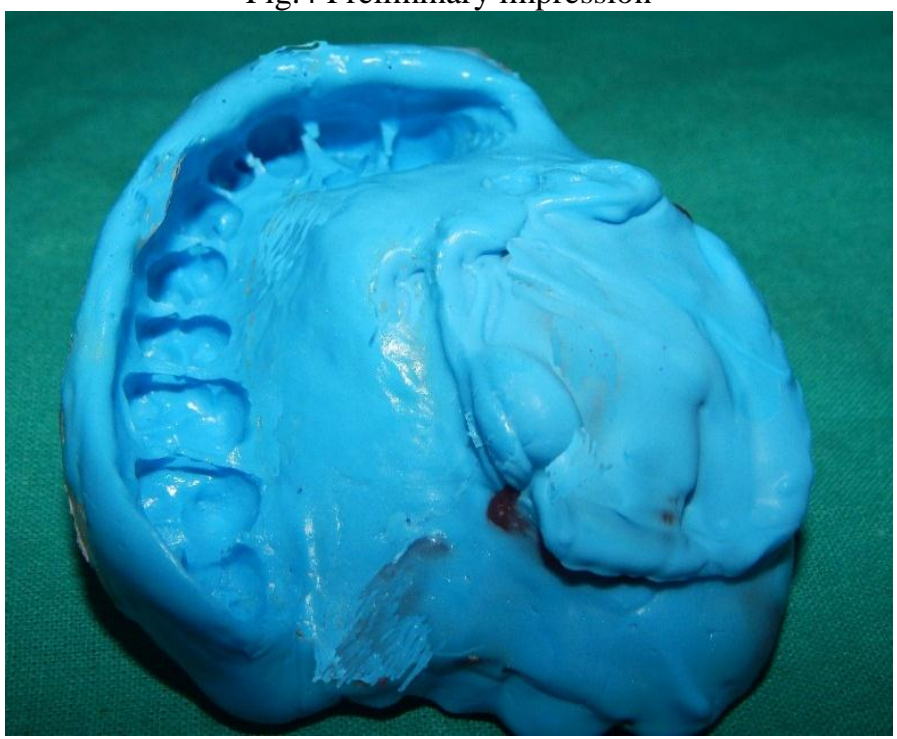

Fig.5 Secondary impression

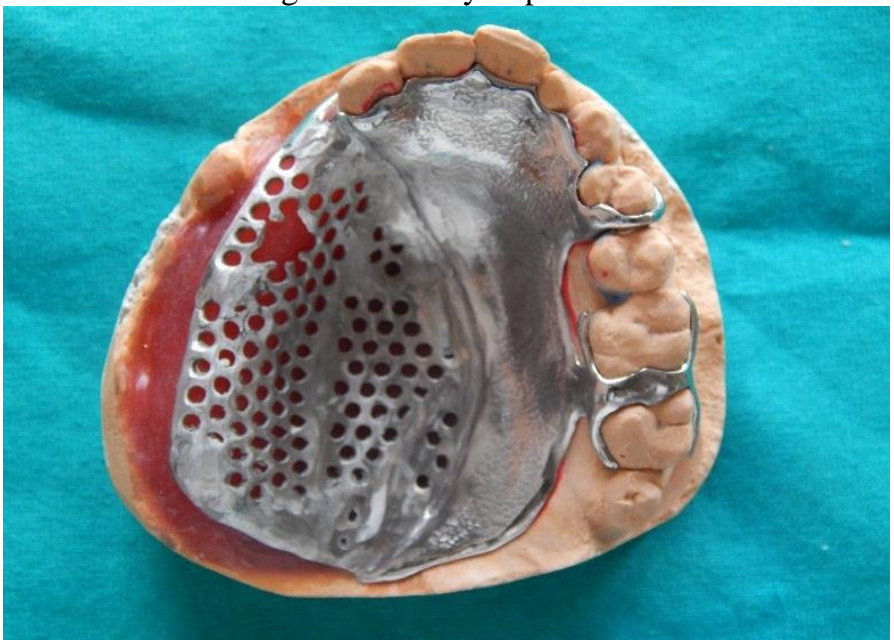

Fig.6 Metal frame work 


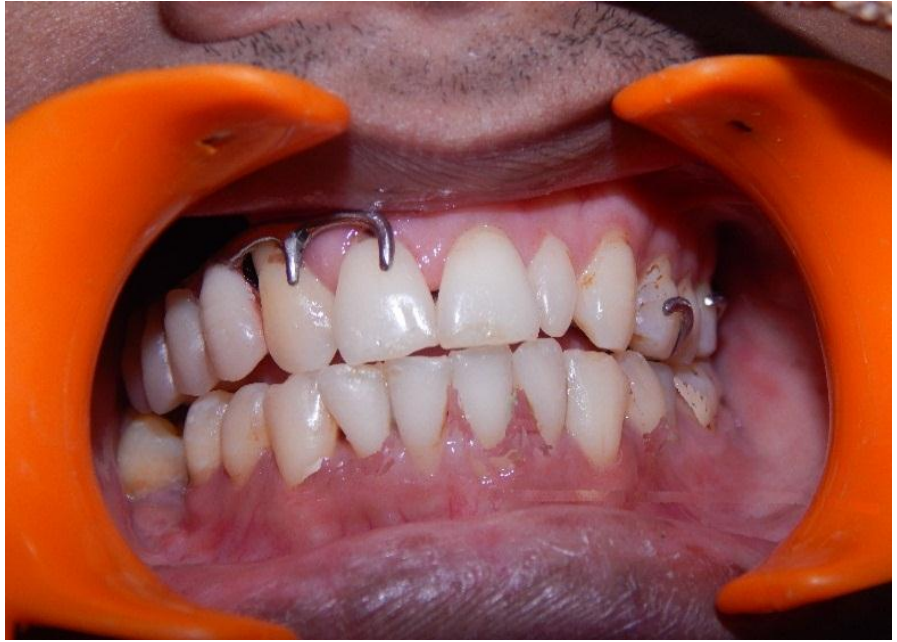

Fig.7 Try in procedure

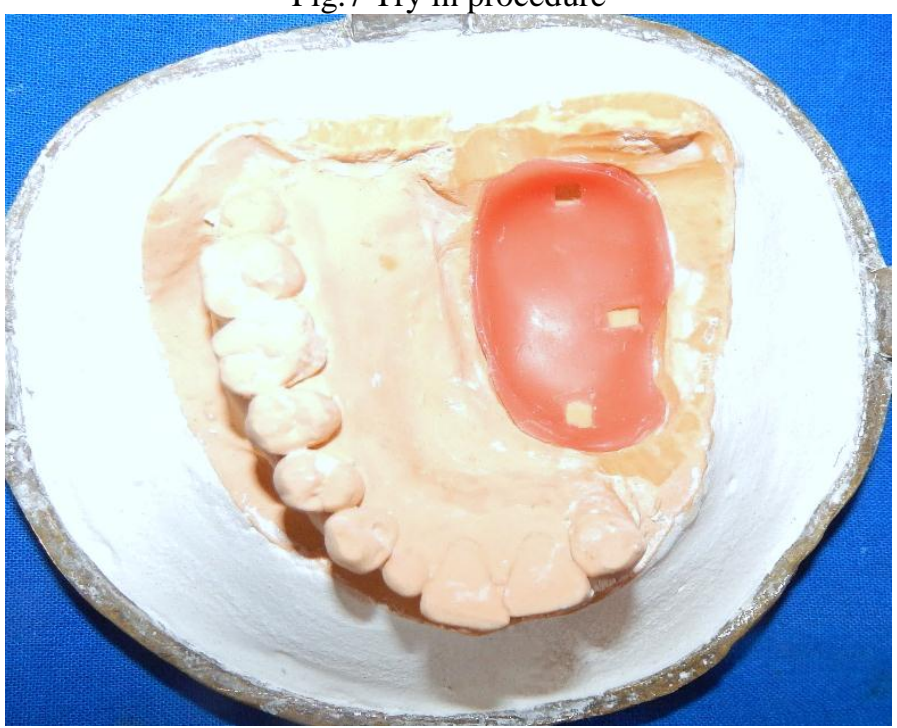

Fig.8 Wax spacer with stoppers

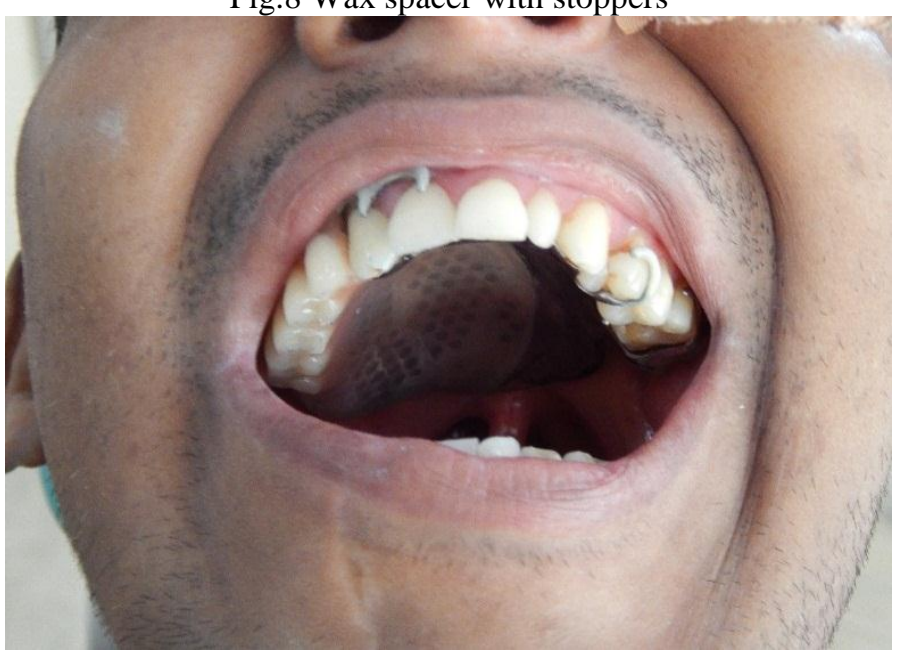

Fig.9 Obturator insertion 


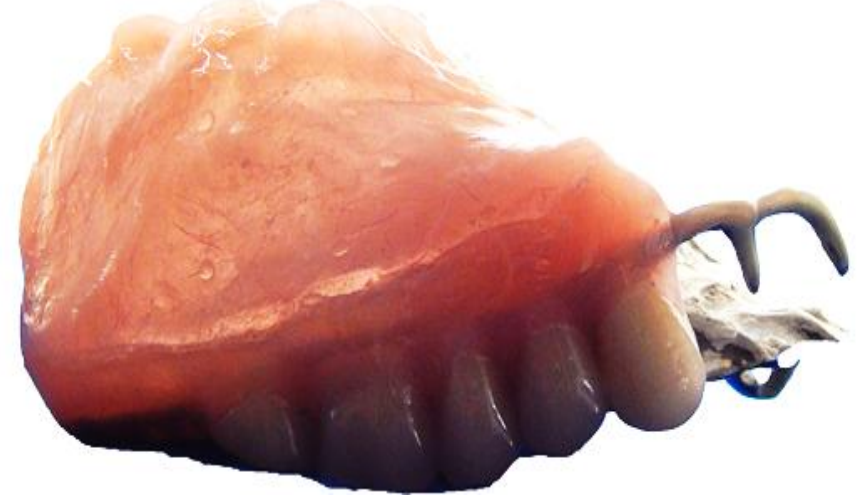

Fig.10 Hollow bulb cast obturator

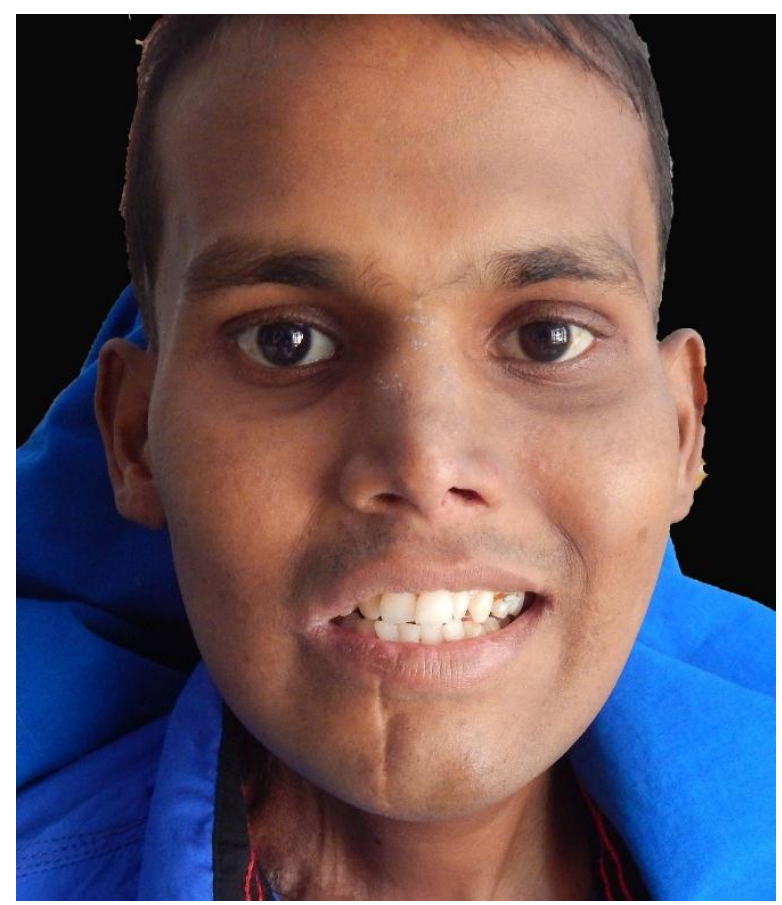

Fig.11 Post operative photograph 